\title{
Density-functionals not based on the electron gas: Local-density approximation for a Luttinger liquid
}

\author{
N. A. Lima, M. F. Silva, and L. N. Oliveira \\ Departamento de Física e Informática, Instituto de Física de São Carlos, Universidade de São Paulo, \\ Caixa Postal 369, 13560-970 São Carlos, SP, Brazil \\ K. Capelle \\ Departamento de Química e Física Molecular, Instituto de Química de São Carlos, Universidade de São Paulo, \\ Caixa Postal 780, São Carlos, 13560-970 SP, Brazil
}

(November 20, 2018)

\begin{abstract}
By shifting the reference system for the local-density approximation (LDA) from the electron gas to other model systems one obtains a new class of density functionals, which by design account for the correlations present in the chosen reference system. This strategy is illustrated by constructing an explicit LDA for the one-dimensional Hubbard model. While the traditional ab initio LDA is based on a Fermi liquid (the electron gas), this one is based on a Luttinger liquid. First applications to inhomogeneous Hubbard models, including one containing a localized impurity, are reported.

71.15.Mb, 71.10.Pm, 71.10.Fd, 71.27.+a
\end{abstract}

Density-functional theory (DFT) [1] is the basis of almost all of todays electronic-structure theory, and much of materials science and quantum chemistry. Many-body effects enter DFT via the exchange-correlation $(x c)$ functional, which is commonly approximated by the localdensity approximation (LDA) [1]. The essence of the LDA is to locally approximate the $x c$ energy of the inhomogeneous system under study by that of the homogeneous electron gas. This electron gas plays the role of a reference system, whose correlations are transfered by the LDA into the DFT description of the inhomogeneous system. The most popular improvement upon the LDA are generalized gradient approximations [2], whose basic philosophy is to abandon the requirement of homogeneity of the reference system. This system, however, is normally still the interacting electron gas [2].

In the present paper we propose to explore a different paradigm for the construction of novel density functionals: instead of sticking to the electron gas as a reference system, and abandoning homogeneity, it may sometimes be advantageous to do the reverse: stick to homogeneity (and thus to the LDA) but abandon the electron gas as a reference system. The new reference system is chosen such that it accounts for the correlations present in the inhomogeneous system under study.

The only requirement for the reference system is that in the absence of any spatially varying external potential its $x c$ energy must be known exactly or to a high degree of numerical precision. Besides the electron gas (or Jellium model) there are many other physically interesting model systems that satisfy this criterium. Most notably among these is a large class of low-dimensional models which can be solved exactly by Bethe Ansatz (BA) techniques or bosonisation (in one dimension, e.g., the repulsive and the attractive Hubbard model, the hard-core Fermi and
Bose gases, the Heisenberg, the supersymmetric t-J, and the Tomonaga-Luttinger model $[3,4]$ ). The solutions to these models in the homogeneous case can be used instead of the electron gas to construct LDA functionals that can then be applied to study these models also in inhomogeneous situations. The main advantage offered by a DFT treatment of such models is the gain in simplicity that arises from mapping the inhomogeneous interacting many-body system onto a noninteracting auxiliary system, which is diagonalized much more easily.

Below we implement these ideas for the onedimensional Hubbard model (1DHM), by constructing an LDA based on the exact Bethe Ansatz solution of Lieb and Wu [5]. A DFT treatment of the Hubbard model has been pioneered by Gunnarsson and Schönhammer in Ref. [6], but the LDA-type functional they proposed has in practice often led to disapointing results [7], and was criticized as not being a proper LDA since it was not based on the exact solution of a homogeneous reference system [8]. As a consequence, more complicated approximation schemes, such as self-interaction corrections, are often employed [9]. An attempt to base a proper LDA for the Hubbard model on the BA was made in Ref. [10], but the formulation of that work has not been widely applied, probably because no explicit expression for the resulting $x c$ functional was provided.

Motivated by our above analysis of the possibility of switching reference systems for the LDA we construct, in the present paper, an explicit and simple BA $x c$ functional and apply it to a variety of inhomogeneous Hubbard models, among them an impurity model for previously unattainable system sizes. This functional, denoted the BA-LDA, has built into it the Luttinger-liquid correlations present in the 1DHM [3,4], in the same way in which the conventional LDA has built into it the Fermi- 
liquid correlations present in the electron gas.

The Hamiltonian for the homogeneous 1DHM is, in standard notation,

$$
\hat{H}=-t \sum_{\langle i j\rangle, \sigma} c_{i \sigma}^{\dagger} c_{j \sigma}+U \sum_{i} c_{i \uparrow}^{\dagger} c_{i \uparrow} c_{i \downarrow}^{\dagger} c_{i \downarrow} .
$$

Here $t$ is the kinetic energy (in the following taken to be the unit of energy) and $U$ the interaction (considered a fixed parameter, characterizing the Hamiltonian). To construct an LDA we first develop a parametrization for the total energy per site, as a function of $U$ and $n$ (the filling factor, a constant in the homogeneous case). Our parametrization interpolates analytically between three limiting cases in which explicit results can be extracted from the Bethe-Ansatz solution [3,5]: (i) $U \rightarrow 0$ and any $n \leq 1$, (ii) $U \rightarrow \infty$ and any $n \leq 1$, and (iii) $n=1$ and any $U[11]$. The expression

$$
e^{B A}(n, U)=-\frac{2 \beta(U)}{\pi} \sin \left(\frac{\pi n}{\beta(U)}\right),
$$

where $\beta(U)$ is an ( $n$ independent) number which is determined for any given value of $U$ from

$$
-\frac{2 \beta}{\pi} \sin \left(\frac{\pi}{\beta}\right)=-4 \int_{0}^{\infty} d x \frac{J_{0}(x) J_{1}(x)}{x[1+\exp (U x / 2)]},
$$

and $J_{0}$ and $J_{1}$ are zero and first order Bessel functions, recovers all three limits: The right-hand side of Eq. (3) is the exact BA expression for the total energy at half filling. The parameter $\beta$ is thus determined such that at $n=1 \mathrm{Eq}$. (2) becomes exact. On the other hand, Eq. (2) is already of the algebraic form of the exact results for the limits $U=0$ and $U \rightarrow \infty$, in which $\beta=2$ and $\beta=1$, respectively. In these limits the integral in Eq. (3) can be calculated analytically, and one indeed recovers these values for $\beta(U)$ [12].

Eq. (2) with $\beta(U)$ determined from (3) is thus exact in the three situations mentioned above. In order to check whether these equations provide a reasonable approximation also between these limits, we have numerically solved the Lieb-Wu integral equations for the full Bethe Ansatz solution [5] and compared the resulting total and $x c$ energies with the one obtained from Eqs. (2) and (3). We find that both agree to within at most a few percent, even for values of the parameters far away from the exact limiting cases. This is illustrated in the inset of Fig. 1.

In order to extract the exchange-correlation energy $e_{x c}^{B A}(n, U)$ from the total-energy expression (2), one follows the usual prescription of DFT [1] and subtracts the Hartree energy and the noninteracting kinetic energy. This latter energy is simply given by substituting $\beta=2$ in Eq. (2). In the spirit of the usual electron gas LDA we can then construct an LDA for the 1DHM, i.e.,

$$
E_{x c}^{B A-L D A}\left[n_{i} ; U\right]=\left.\sum_{i} e_{x c}^{B A}(n, U)\right|_{n \rightarrow n_{i}},
$$

where $n_{i}=\sum_{\sigma}\left\langle c_{i \sigma}^{\dagger} c_{i \sigma}\right\rangle$. Given this expression for the $x c$ functional, ground-state properties of Hubbard models subject to a wide spectrum of inhomogeneities can be calculated from DFT. Note that in such a calculation Eq. (3) must be solved only once for any given value of $U$, i.e., determination of $\beta$ takes place outside the selfconsistency cycle of DFT.

As a first numerical example we apply the BA-LDA to a finite 1DHM with open boundary conditions, and calculate its ground-state energy as a function of the number of sites $L$. In Fig. 1 we compare our results with those obtained from exact (Lanczos) diagonalization of the same system. We see that around $L=7$ there is a crossover between exact and approximate data points (separately for even and odd values of $L$ ). After that, the deviation between both sets of data saturates to a value near the intrinsic error of the interpolation (2), indicated by the error bar. As an example for a truly inhomogeneous system we now add an on-site potential $\sum_{i \sigma} v_{i} c_{i \sigma}^{\dagger} c_{i \sigma}$ to the Hamiltonian. Our results for a binary potential (with $v_{i}=-1$ on the odd sites and $v_{i}=+1$ on the even ones) are displayed and compared with exact diagonalization in Fig. 2.

We have performed similar calculations also for several other values of $U$ and other external potentials $v_{i}$. Our conclusions from these calculations are: (i) The accuracy of the BA-LDA total energy is typically of the order of a few percent, and much better than that near crossovers and near the limits at which equation (2) exactly represents the underlying homogeneous reference data $(U=0, U \rightarrow \infty, n=1)$. (ii) Unlike traditional methods, the quality of the BA-LDA does not deteriorate as $L$ increases, and the computational effort associated with it is that of diagonalizing a noninteracting system. Fully self-consistent calculations for systems with tens of thousands of sites are thus possible. This is a unique, and rather desirable, feature of the BA-DFT, as compared to traditional methods.

Interestingly, when one of the site occupation numbers comes very close to 1 (typically within less than $5 \times 10^{-3}$ ), the self-consistency cycle associated with the Hubbard model Kohn-Sham equations does not necessarily converge. In the homogeneous case $n=1$ (half filling) marks the Mott metal-insulator transition associated with the opening of a gap in the energy spectrum [3-5]. Whenever in a metallic system one of the site occupation numbers comes close to 1 , a local approximation, such as the BALDA, thus treats the system at that site as if it were an insulator, in spite of the fact that the metallic (Luttinger liquid) correlations are very different from those of the Mott insulator. The 1DHM thus constitutes a theoretical laboratory in which the band-gap problem of DFT can be studied $[6,10]$. Results obtained with the present functional will be reported in a forthcoming publication.

After these preliminary investigations we now consider a case that illustrates the full power of the BA-LDA ap- 
proach: a localized impurity. For this kind of system traditional approaches face the problem of slow convergence to the thermodynamic limit. Different types of impurities in the Hubbard model have been studied in the literature by various techniques $[6,13]$. Here we model the impurity by choosing $v_{I}=-1$ at the impurity site and $v=0$ everywhere else, so that electrons will be dragged to the impurity site. The density distribution for the 201-site system is displayed in Fig. 3, while the convergence to the thermodynamic limit is illustrated in Fig. 4, which also contains results obtained for a much more attractive impurity with $v_{I}=-10$.

The difference between open and periodic boundary conditions becomes small only when the system size $L$ exceeds the damping length of the Friedel oscillations originating at the surface in the open case. To bring out clearly the effect of the impurity, regardless of the choice of boundary conditions, we have, in both figures, subtracted the results for the same boundary conditions without the impurity. What remains are the Friedel oscillations originating at the impurity. These oscillations on their own significantly slow down the convergence of the total energy to the thermodynamic limit: Fig. 4 shows that, as expected in the thermodynamic limit, the impurity-added contribution to the total energy scales linearly with the impurity concentration $1 / L$. The impurity problem thus illustrates an area in which the BALDA can be useful, since systems of the size required to approximate the thermodynamic limit to within a percent or better are hard to study with traditional methods, in particular for periodic boundary conditions.

For small systems, where exact diagonalization is possible, we also compared the density distributions obtained from the BA-LDA with the exact ones. Both agree quantitatively. For larger systems one can use the BALDA results to study the asymptotic algebraic decay of the oscillations. For the case depicted in Fig. 3 we find, for example, that the oscillations decay as $1 / x^{\gamma}$, where $x$ is the distance to the impurity site and $\gamma(U=6)=1.30$. This exponent is a nonuniversal (interaction-dependent) parameter characteristic for the impurity system. By repeating this calculation for other values of $U$ we obtain, e.g., $\gamma(U=4)=1.25, \gamma(U=2)=1.20$, and $\gamma(U=0)=1.0$.

For the BA-LDA the limit on the size of the systems which can be treated is much less restraining than for traditional methods, since one must diagonalize only a noninteracting (Kohn-Sham) Hamiltonian. For small systems the accuracy attained is clearly inferior to densitymatrix renormalization group [14] or Quantum Monte Carlo [15] methods. This situation parallels the one in which DFT finds itself in ab initio calculations: Practical applications of $a b$ initio DFT usually do not lead to high accuracy. Band structures, for example, are more accurately calculated using GW [16], and properties of small molecules are better obtained from CI [17]. However, these are computationally expensive methods that place great demands on ones resources and are not easily applicable to complex and/or large systems (e.g. molecules with more than a few atoms). The power of $a b$ initio DFT arises from the relative facility with which it is applied to large and complex systems. In the same spirit, DFT using generalized LDA's may provide a useful alternative to traditional methods for correlated models with a large number of sites. In order to further explore these possibilities, we are currently applying the BA-LDA to the Mott insulating phase of the 1DHM and to a more detailed study of the Friedel oscillations around an impurity. Results will be reported in forthcoming publications.

Acknowledgments This work was supported by FAPESP and CAPES. KC thanks A. J. R. da Silva for valuable discussions.

[1] P. Hohenberg and W. Kohn, Phys. Rev. 136, B864 (1964). W. Kohn and L. J. Sham, Phys. Rev. 140, A1133 (1965). W. Kohn, Rev. Mod. Phys. 71, 1253 (1999).

[2] J. P. Perdew, K. Burke, and M. Ernzerhof, Phys. Rev. Lett. 77, 3865 (1996).

[3] P. Schlottmann, Int. J. Mod. Phys. B 11, 355 (1997).

[4] J. Voit, Rep. Prog. Phys. 58, 977 (1995).

[5] H. A. Bethe, Z. Phys. 71205 (1931). E. H. Lieb and F. Y. Wu, Phys. Rev. Lett. 20, 1445 (1968). Y. Ogata and H. Shiba, Phys. Rev. B 41, 2326 (1990). H. J. Schulz, Phys. Rev. Lett. 64, 2831 (1990).

[6] O. Gunnarsson and K. Schönhammer, Phys. Rev. Lett. 56, 1968 (1986).

[7] E. Runge and G. Zwicknagl, Ann. der Physik 5, 333 (1996).

[8] L. J. Sham and M. Schluter, Phys. Rev. Lett. 60, 1582 (1988).

[9] A. Svane and O. Gunnarsson, Phys. Rev. B 37, 9919 (1988). J. A. Majewski and P. Vogl, Phys. Rev. B 46, 12219 (1992). Y. Xie, R. Han, and X. Zhang, Phys. Rev. B 58, 12721 (1998).

[10] K. Schönhammer, O. Gunnarsson, and R. M. Noack, Phys. Rev. B 52, 2504 (1995).

[11] The extension to a more than half-filled band $(n>1)$ is accomplished by a particle-hole transformation [5].

[12] In general, Eq. (3) can have more than one solution for $\beta$ for a given $U$. When this happens we always choose the one between the two analytical solutions 1 and 2, corresponding to the two extreme values of $U$.

[13] G. Bedürftig et al., Phys. Rev. B 58, 10225 (1998). A. G. Rojo, R. Allub, and M. C. G. Passeggi, Phys. Rev. 39, 2685 (1989). A. A. Zvyagin and P. Schlottmann, Phys. Rev. B 56, 300 (1997). P. Seb and A. Singh, Phys. Rev. B 53, 328 (1996).

[14] S. R. White, Phys. Rev. Lett. 69, 2863 (1992). ibid 77, 3633 (1993). 
[15] W. von der Linden, Phys. Rep. 220, 53 (1992).

[16] F. Aryatesiawan and O. Gunnarsson, Rep. Prog. Phys. 61, 237 (1998).

[17] A. Szabo and N. S. Ostlund, Modern Quantum Chemistry (McGraw-Hill, New York, 1989).

FIG. 1. Main figure: Total energy calculated from BA-LDA (open squares) and exact diagonalization (full circles) versus system size, for a $1 \mathrm{DHM}$ with open boundary conditions and $U=6$. For even $L$ we take $N=L / 2$ (so that $n=N / L=1 / 2$, corresponding to quarter filling). For odd $L$ we take $N=(L-1) / 2$. The error bar at $L=14$ represents the intrinsic error of the parametrization (2), as estimated from the inset. For $L=14$ the BA-LDA data agree with the exact ones within this error. Inset: Exchange-correlation energy of the infinite 1DHM as a function of $n$ for several values of $U$. The full curves represent our parametrization (2) with (3), and the symbols represent values obtained from numerical solution of the Lieb-Wu BA integral equation for $U=3,6$, and 9. For $U=0$ and $U=\infty$ the parametrization is exact.

FIG. 2. Main figure: Total energy of the 1DHM with a binary site potential and periodic boundary conditions, as described in the main text, for $U=6$ and $n=1 / 2$. Open squares: BA-LDA, full circles: exact diagonalization. Inset: exact $x c$ energy and LDA $x c$ energy, evaluated at exact and LDA densities, respectively. The absolute errors in the LDA $x c$ energy and total energy are different because in the LDA the Hartree, external potential and noninteracting kinetic components of the total energy are also evaluated at the LDA density, not at the exact one. However, the $x c$ energy is the dominating source of error in the total energy and the relative error in the total energy is considerably smaller than that in its $x c$ component.

FIG. 3. Density distribution for a system with $L=201$ sites, $N=101$ electrons, $U=6$, and a localized impurity with $v_{i m p}=-1$. Main curve: periodic boundary conditions. Inset: open boundary conditions. The density of the same system without impurity has been subtracted to display clearly the impurity-added contribution.

FIG. 4. Total energy calculated from BA-LDA for the impurity model of Fig. 3, with open (full symbols) and periodic (empty symbols) boundary conditions, plotted as a function of the inverse system size. Impurity strength $v_{I}=-1$ (upper curve), and $v_{I}=-10$ (lower curve). 


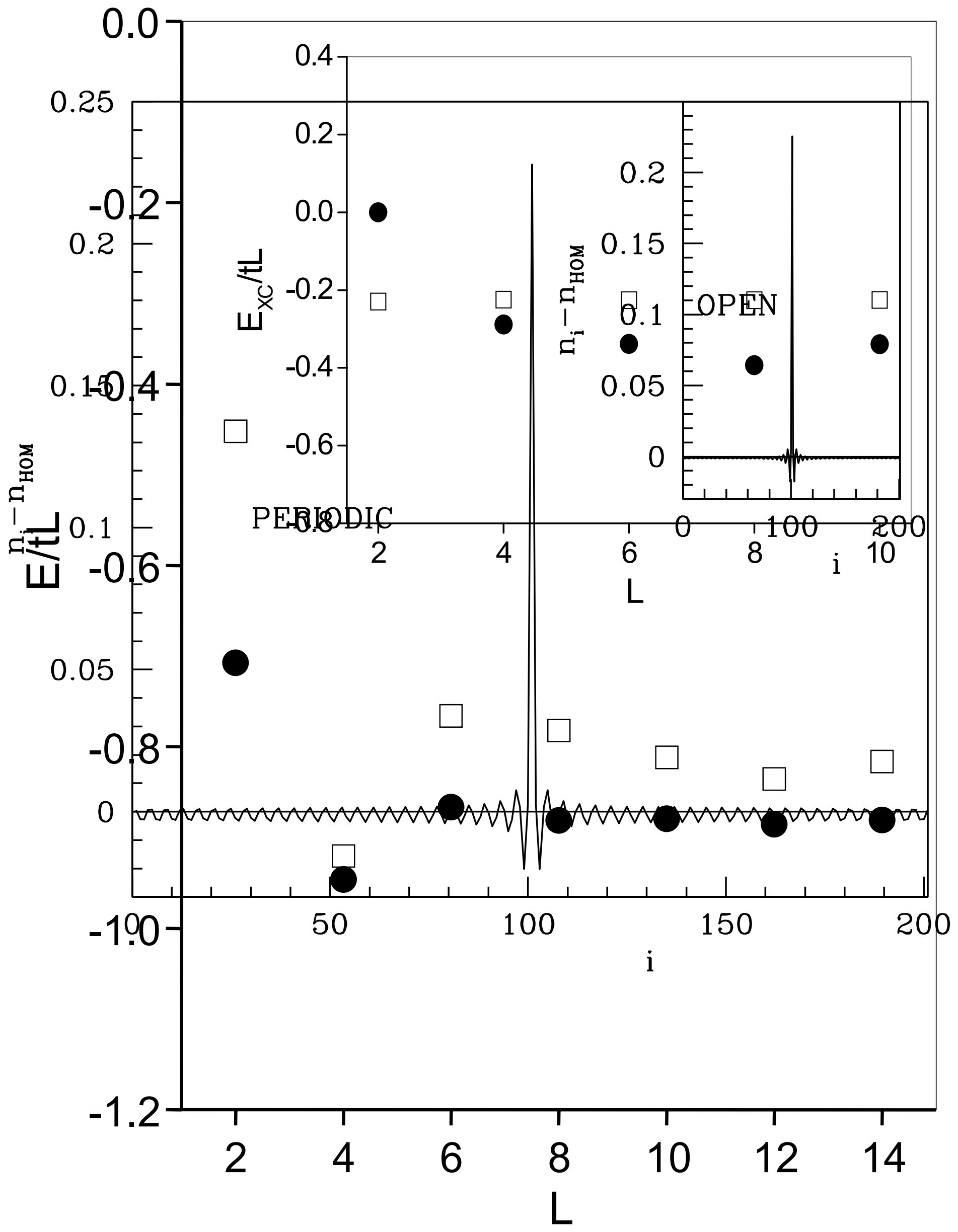

